\title{
Modelo experimental de sutura manual em colon de cão por vídeo-laparoscopia ${ }^{1}$
}

\author{
Experimental model of laparoscopic handsewn suture in colon of dogs
}

\author{
Sthela Maria Murad Regadas ${ }^{2}$, Francisco Sérgio P. Regadas ${ }^{3}$, Lusmar Veras Rodrigues ${ }^{4}$, Mauricio C.G.S. Carvalho ${ }^{5}$, \\ Francisco Sérgio P. Regadas Filho ${ }^{6}$ \\ 1. Trabalho apresentado ao Curso de pós-Graduação do Departamento de Cirurgia da Faculdade de Medicina da Universidade Federal do Ceará \\ para obtenção do grau de Mestre em Técnica Operatória e Cirurgia Experimental. \\ 2. Mestre em Técnica Operatória e Cirurgia Experimental pela Faculdade de Medicina da U.F.C. Pós-graduanda (nível de Doutorado) da \\ Faculdade de Medicina da U.F.C. \\ 3. Professor Titular e Coordenador da Disciplina de Cirurgia Digestiva da Faculdade de Medicina da U.F.C. Diretor da Unidade de Serviços \\ Cirúrgicos do Hospital das Clínicas da U.F.C. \\ 4. Professor Livre Docente, Chefe do Departamento de Cirurgia da Faculdade de Medicina da U.F.C. \\ 5. Residente (R3) do Serviço de Cirurgia Geral do Hospital das Clínicas da U.F.C. \\ 6. Aluno de Graduação da Faculdade de Medicina da U.F.C. \\ 7. Aluno de Graduação da Faculdade de Medicina da Universidade Católica de Brasília
}

\section{RESUMO}

Objetivo: Desenvolver um modelo experimental de endo-sutura manual laparoscópica em cólon destinado principalmente ao treinamento de cirurgiões. Métodos: Foram operados 40 cães mestiços, machos, com peso entre 15 e 20 kg, provenientes do laboratório da Faculdade de Veterinária da Universidade Estadual do Ceará. Foram distribuídos aleatoriamente em dois grupos de 20 animais cada: GI-secção e hemostasia do colon com bisturi elétrico e GII-secção do colon com tesoura. Cada grupo foi subdividido em dois subgrupos com 10 animais cada, conforme o acesso abdominal utilizado - A-acesso laparotômico e B-laparoscópico. Sob anestesia geral endovenosa, foi realizada incisão transversal no cólon sigmóide, numa extensão de $50 \%$ da circunferência, distando $15 \mathrm{~cm}$ da reflexão peritoneal, sendo em seguida suturada em plano único, extra-mucoso, com pontos separados de fio de polidioxanona 000 (PDSß). Os animais foram avaliados quanto à evolução clínica, análise macroscópica, teste de tensão da sutura e estudo histológico qualitativo. Foram sacrificados no $7^{\circ}$ dia de pós-operatório. Para analise estatística, foram utilizados os testes Qui-quadrado corrigido de Yates e o teste exato de Fisher. Resultados: Todos os animais cuja colotomia foi realizada com tesoura (GIIA IIB) e 9 operados com bísturi elétrico (GI), sendo 5 (50,0\%) operados pelo acesso laparotômico (GIA) e 4 (40,0\%) pelo laparoscópico (GIB), apresentaram evolução clínica satisfatória, deambulando e aceitando bem a dieta oral a partir do primeiro dia de pós-operatório. A primeira evacuação ocorreu entre 48 a 72 horas de pós-operatório. Não apresentaram diarréia nem vômitos. Onze animais do grupo GI, sendo 5 (50\%) do subgrupo IA e 6 (60,0\%) do IB não aceitaram bem a dieta oral, apresentando diarréia (3 a 5 evacuações líquidas por dia) e vômitos (1 a 3 episódios por dia) evoluindo para óbito entre o quarto e o sétimo dia do pós-operatório. Comparando os grupos GI com GII, foi observada diferença significante $(\mathrm{p}<0,005)$, sem no entanto observarse diferença quando comparados os subgrupos IA e IB. A sutura permaneceu íntegra em todos (100,0\%) os animais GII e em 5 GI, demonstrando portanto diferença estatisticamente significante $(\mathrm{p}<0,005)$. Desses, $3(30,0 \%)$ eram do subgrupo IA e 2 (20,0\%) do IB. Foi evidenciada sutura bloqueada com epíplon em quatro animais, sendo dois (20\%) do subgrupo IA e dois (20\%) do IB. Houve deiscência da sutura com peritonite em 11 (55\%) animais do grupo I (p<0,005), sendo 5 (50\%) do subgrupo IA e 6 (60\%) do IB (p>0,005). Todos evoluíram para óbito entre o quarto e o sétimo d.p.o. O teste de tensão da sutura foi realizado com a pressão média de 222,1 mmHg e não ocorreu ruptura do cólon em nenhum dos animais do grupo II e em 5 (40,0\% ) do Grupo I ( $\mathrm{p}<0,005)$. Desses, 3 (30\%) eram do subgrupo IA, e 2 (20,0\%) do IB (P>0,005). Ocorreu ruptura da sutura em 4 ( $20 \%$ ) cães do grupo I, sendo 2 do subgrupo IA e 2 (20\%) do IB com pressão média de 94,0 mmHg. A análise histológica das amostras obtidas no $7^{\circ}$ d.p.o. demonstrou reprodução das fases do processo inflamação-reparação em ambos acessos utilizados. Conclusão: A endo-sutura manual laparoscópica em cólon de cães pode ser utilizada com segurança pois apresentou os mesmos resultados do acesso laparotômico. Os resultados dependem especialmente do treinamento adequado do cirurgião em técnicas cirúrgicas laparoscópicas.

Descritores: Sutura. Colon. Laparoscopia.

\section{ABSTRACT}

Purpose: Develop an experimental model of laparoscopic hand-sewn suture in colon of dogs to be specially used for surgeons' trainning. Methods: Forty male dogs were operated on, weight between 15 and $20 \mathrm{~kg}$, from the laboratory of the Veterinary School of the State University of Ceara. They were distributed within two groups with 20 animals each: GI- The colonic wall incision was done with an electrical scapel followed by haemosthasia and GII- The colonic wall incision was 
performed with a scissors. Each group was shared in two other groups with 10 animals each, according to the abdominal approach used. A- Laparotomic approach and B-Laparoscopic approach. Under intra-venous general anesthesia, a transverse incision was performed envolving $50 \%$ of the sigmoid wall, $15 \mathrm{~cm}$ far from the pelvic peritoneal pouch, followed by an extramucosa, one layer and interrupted suture with 000 polidioxanona (PDSâ). The animals were evaluated concerning to the clinical recovery, macroscopic appearance, suture-tension test and histologic study. The animals were sacrified on the 7th postoperative day. Fisher and Qui-square tests were used for statistical analysis. Results: All the animals submitted to colotomy performed by scissor (GIIA, GIIB) and nine animals operated on with an electrical scapel (GI), five (50,0\%) by laparotomic approach (GIA) and four (40,0\%) laparoscopically (GIB) had a satisfactory clinical recovery, walking and accepting oral diet on the first post-operative day. The first bowel movement occurred between 48 and 72 hours. It didn’t occur diarrhea or vomits. Eleven animals from group I, five (50\%) from group IA and six $(60,0 \%)$ from group IB didn't accept oral diet and complained of diarrhea (3 to 5 evacuations each day), 1 to 3 episodes of vomits each day and they die between the fourth and seventh postoperative day. Comparing groups GI with GII, a statistical significant difference was observed $(\mathrm{p}<0,005)$. There was no difference between GIA and GIB. Colonic sutures were intact in all the animals from group II and in five from group I with statistical significant difference $(\mathrm{p}<0,005)$. Three $(30,0 \%)$ were from group IA and two $(20,0 \%)$ from group IB. The colonic suture was envolved by epiplon in four animals, two (20\%) from GIA and 2 (20\%) from GIB. Suture dehiscence with peritonitis occurred in eleven (55\%) animals from GI ( $<<0,005)$, five (50\%) from GIA and six (60\%) from GIB $(p>0,005)$. All the animals died between the fourth and seventh postoperative day. The tension suture test was performed with an average pressure of 222,1 mmHg and there was no colonic suture disruption in any animal from the group II and in five (40,0\%) from the group I ( $<<0,005)$. Three (30\%) animals were from GIA and two $(20,0 \%)$ from GIB (P>0,005). Colonic suture rupture occurred in four (20\%) dogs from group I, two from GIA and two (20\%) from GIB with an average pressure of $94,0 \mathrm{mmHg}$. The histological analysis of the surgical specimens removed on the 7 th postoperative day demonstrated the same level of the inflamatory process in both approach used. Conclusion: The handsewn laparoscopic colonic suture in dogs can be safely performed, showing the same results of the laparotomic approach. The surgical results depend specially on the adequate surgeon's trainning in laparoscopic surgical technique.

Key words: Colon. Suture. Laparoscopy.

\section{Introdução}

Os primeiros procedimentos colorretais laparoscopicos foram realizados em 19901-9. No Brasil, Regadas e col. Iniciaram no ano seguinte, executando reconstituição de trânsito intestinal após procedimento de Hartmann em quatro pacientes ${ }^{10}$. A utilização do acesso laparoscópico no tratamento de diversas afecções abdominais resultou no desenvolvimento de aparelhos, instrumentais cirúrgicos e também de técnicas de endo-sutura manual e mecânica. Para tanto, foram realizados vários estudos experimentais, visando desenvolver essas técnicas de endo-sutura manual, principalmente em intestino delgado e colon ${ }^{11-15}$. Para isso, fez-se necessário treinamento adequado e específico associando habilidade manual, coordenação motora e visual $^{16}$. Apesar do desenvolvimento de instrumental apropriado para realização de anastomoses intraperitoneais por vídeolaparoscopia, existem situações em que a endosutura manual faz-se necessária, como na retopexia, nas rafias de lesão acidental de intestino delgado, cólon, bexiga ou ureter, na excisão de tumores benignos por colotomia, na síntese de lesão acidental do coto retal e muitas outras, que poderiam tornar necessária a conversão do procedimento laparoscópico em laparotômico. O objetivo deste estudo é desenvolver um modelo experimental de endo-sutura manual laparoscópica em cólon destinado principalmente ao treinamento de cirurgiões.

\section{Métodos}

Foram utilizados 40 cães mestiços, machos, com peso entre 15 e $20 \mathrm{~kg}$, clinicamente sadios, oriundos do canil da Prefeitura Municipal de Fortaleza, Estado do Ceará e vacinados contra raiva. Os animais foram mantidos no canil do laboratório de Cirurgia Experimental da Universidade Estadual do Ceará sob condições adequadas de umidade e temperatura recebendo água e ração ad libitum e cuidados gerais conforme os preceitos do Guia de Cuidados e Utilização dos Animais de Laboratório do "US Department of Health and Human Services”, 1985. Os cães foram distribuídos aleatoriamente em dois grupos de 20 animais cada. Todos foram submetidos à secção do cólon esquerdo, numa extensão aproximadamente de $50 \%$ da circunferência. Em seguida, foi realizada sutura manual, extra-mucosa, com pontos separados utilizando fio de polidioxanona 000. No grupo I (GI), foi realizada secção e hemostasia de todas as camadas do cólon com bisturi elétrico; no grupo II (GII), a secção das camadas foi realizada com tesoura e a hemostasia com gaze cirúrgica. Cada grupo foi subdividido em dois subgrupos com 10 animais cada, conforme o acesso abdominal utilizado: Subgrupo A, quando o acesso foi laparotômico (A) e subgrupo B, quando o acesso foi laparoscópico (B). Todos os cães tiveram ingesta de água à vontade nas 24 horas que antecederam a intervenção cirúrgica. Todos foram submetidos a clister evacuativo com $120 \mathrm{ml}$ de solução glicerinada a 10\%, às 12 e 2 horas antes do procedimento cirúrgico. Os animais foram submetidos à anestesia geral endovenosa, utilizando-se xilasina, midazolam e ketamina, sendo $1 \mathrm{ml}$ de cada substância diluido em $7 \mathrm{ml}$ de soro fisiológico, na dose de $4 \mathrm{ml} / \mathrm{Kg}$ de peso. Não foi necessária entubação endo-traqueal.

Técnica Operatória - Acesso laparotômico - O animal era posicionado e imobilizado em decúbito dorsal sobre mesa cirúrgica adequada. Em seguida, era feita antissepsia da parede abdominal anterior com iodopovidine e assepsia do campo operatório. Posteriormente, era realizada incisão mediana de $6 \mathrm{~cm}$, distal a genitalia. Após abertura da cavidade abdominal, o cólon esquerdo era identificado e 
apreendido com dois clampes intestinais, sendo exposta sua borda antimesentérica, que era seccionada transversalmente, numa extensão de 50\% da circunferência, distando $15 \mathrm{~cm}$ da reflexão peritoneal. Utilizaram-se bisturi elétrico (GIA) ou tesoura com hemostasia por compressão com gaze cirúrgica (GIIA). Acesso laparoscópico - Foram utilizados monitor colorido, de 20 polegadas, 720 linhas de definição; processador de imagem digital de 1,5 lux de sensibilidade; fonte de luz Xenon de 300 w de potência; cabo ótico de lente condensada; videocassete de alta sensibilidade; ótica zero grau (10 mm) e insuflador de ar. O insuflador de ar para pneumoperitônio era constituído por uma bomba insufladora (mini-compressor) e três tubos de polietileno, conectados em forma de "Y" e munidos de uma válvula anti-refluxo (válvula própria para esfigmomanômetro). Um dos tubos era conectado a um trocarte, enquanto o outro era ligado a um sistema de coluna líquida em selo d’água do tipo usada em drenagem torácica, de modo a manter a pressão pré-estabelecida ${ }^{17}$ ( Sena 1999). O animal era posicionado em decúbito dorsal, com inclinação lateral direita. O pneumoperitonio era realizado por insuflação de CO2, após punção com agulha de Verres, no flanco direito. Em seguida, foram inseridos cinco trocartes. Um (10 mm) no flanco direito para a ótica, dois outros na fossa iliaca direita $(10 \mathrm{~mm})$ e epigástrio $(5 \mathrm{~mm})$, à esquerda da linha média para os instrumentos de trabalho de modo que o porta-agulha formasse um ângulo de $90^{\circ} \mathrm{com}$ a pinça auxiliar. As duas outras punções eram feitas no flanco e fossa iliaca esquerda (5 mm) para apreensão e exposição do cólon esquerdo. O cirurgião e o primeiro auxiliar (câmera) posicionaram-se à direita e o segundo auxiliar à esquerda do animal. Em seguida, o cólon esquerdo era identificado e apreendido com duas pinças tipo Babcock, expondo-se a borda antimesentérica e seccionando-o na extensão de 50\% de sua circunferência, utilizando-se bisturi elétrico (GIB) e tesoura com hemostasia por compressão com gaze cirúrgica (GIIB). A sutura foi realizada em plano único, extra-mucosa, com pontos separados, utilizando fio absorvível de polidioxanona 000. Os animais permaneceram em observação clínica recebendo ração e água ad libitum durante sete dias, quando eram submetidos à re-laparotomia exploradora para avaliação da sutura colônica.

Avaliação clínica - Os animais foram avaliados diariamente quanto à aceitação da dieta, ao tipo e número de evacuações e ocorrência de vômitos.

Avaliação da sutura - As suturas foram avaliadas durante a re-laparotomia através dos seguintes critérios:

- Macroscopia - Inspeção da cavidade abdominal quanto à presença de aderências, deiscência, fístulas ou abscessos.

- Teste de tensão - O dispositivo utilizado para registrar as pressões era constituído por esfigmomanômetro, pêra com válvula de controle e cateter uretral de polietileno de 6 Fr. A técnica consistiu na ressecção de segmento colônico de $10 \mathrm{~cm}$ de extensão, contendo a sutura na sua porção média. Em seguida, era feito clampeamento de suas extremidades. Logo após, era confeccionada uma bolsa em uma das extremidades, próxima ao clampe, para introdução de cateter, através do qual era insuflado ar. O segmento de cólon foi completamente distendido até alcançar a pressão máxima registrada no esfigmomanômetro ou constatada a ruptura do colon, ocorrendo vazamento de ar. Para se obter o valor da pressão de distensão máxima, o procedimento acima descrito foi realizado no colon de cinco animais normais, constituindo-o como grupo controle. Ficou estabelecido que a pressão de distensão máxima foi registrada quando o colon se encontrava completamente distendido até ocorrer vazamento do ar através da sutura em bolsa em uma das extremidades. A pressão máxima variou de 220 a 230 mmHg.Não foi possível realizar teste de tensão nos animais que evoluíram com deiscência da sutura. Após avaliação macroscópica, teste de tensão e a colectomia segmentar $(5 \mathrm{~cm})$, os animais eram sacrificados e as peças colocadas em solução de formalina a $10 \%$ e enviadas para análise histológica. As lâminas foram confeccionadas a partir de amostras obtidas na zona de sutura (ZS) e na zona controle (ZC) que está localizada em área normal de intestino grosso, na distância média de $4,0 \mathrm{~cm}$ da sutura e foi utilizada para referência. Foram analisadas qualitativamente por dois patologistas.

\section{Resultados}

Todos os animais cuja colotomia foi feita por tesoura (GIIA IIB) e 9 operados com bísturi elétrico (GI), sendo 5 (50,0\%) operados pelo acesso laparotômico (GIA) e 4 (40,0\%) pelo laparoscópico (GIB) apresentaram evolução clínica satisfatória, deambulando e aceitando bem a dieta oral a partir do primeiro dia de pós-operatório. A primeira evacuação ocorreu entre 48 a 72 horas de pós-operatório. Não apresentaram diarréia nem vômitos. Entretanto, em 11 animais do grupo GI, sendo 5 (50\%) do subgrupo GIA e 6 $(60,0 \%)$ do GIB não aceitaram bem a dieta oral, apresentando diarréia (3 a 5 evacuações líquidas por dia) e vômitos (1 a 3 episódios por dia) evoluindo para óbito entre o quarto e o sétimo dias do pós-operatório. Comparando os grupos GI com GII, foi observada diferença significante $(p<0,005)$. No entanto, sem diferença entre GIA e GIB. A sutura estava íntegra em todos os animais GII e em 5 GI com diferença significante $(\mathrm{p}<0,005)$. Desses, $3(30,0 \%)$ eram do subgrupo GIA e em 2 (20,0\%) do GIB. Foi evidenciada sutura bloqueada com epíplon em quatro animais, sendo 2 (20\%) do subgrupo GIA e 2 (20\%) do GIB. Houve deiscência da sutura com peritonite em 11 (55\%) animais do grupo I ( $p<0,005)$, sendo 5 (50\%) do subgrupo IA e $6(60 \%)$ do GIB ( $p>0,005)$. Todos evoluíram para óbito entre o quarto e o sétimo dias (Tabelas 1, 2 e 3).

O teste de tensão da sutura foi realizado com a pressão

TABELA 1 - Sutura Íntegra - grupos I e II

\begin{tabular}{lllll}
\hline & \multicolumn{2}{c}{ Grupo I } & \multicolumn{2}{c}{ Grupo II } \\
& A & B & A & B \\
& $7^{0}$ & $7^{0}$ & $7^{0}$ & $7^{\circ}$ \\
\hline Sutura íntegra & 3 & 2 & 10 & 10 \\
Sutura tamponada & 2 & 2 & - & - \\
Deiscência sutura & 5 & 6 & - & - \\
\hline Total & 10 & 10 & 10 & 10
\end{tabular}


TABELA 2 - Percentual de deiscência de sutura nos grupos I e II

\begin{tabular}{|c|c|c|c|c|c|}
\hline \multirow[t]{3}{*}{ Grupo } & \multicolumn{4}{|c|}{ Deiscência de Sutura } & Total \\
\hline & \multicolumn{2}{|c|}{ Presente } & \multicolumn{2}{|c|}{ Ausente } & \\
\hline & No. & $\%$ & No. & $\%$ & No. \% \\
\hline I & 11 & 55 & 9 & 45 & 20 \\
\hline II & - & - & 20 & 100 & 20100 \\
\hline
\end{tabular}

Teste Qui-quadrato corrigido de Yates $(\mathrm{p}<0,005)$

TABELA 3 - Percentual de deiscência de sutura nos grupos IA e IB

\begin{tabular}{lcccccc}
\hline Grupo & \multicolumn{3}{c}{ Deiscência da Sutura } & \multicolumn{2}{l}{ Total } \\
& \multicolumn{2}{c}{ Presente } & \multicolumn{2}{l}{ Ausente } & \multicolumn{2}{l}{$l$} \\
& No. & $\%$ & No. & $\%$ & & No. $\%$ \\
\hline IA & 5 & 50 & 5 & 50 & 10 & 100 \\
B & 6 & 60 & 4 & 40 & 10 & 100 \\
\hline
\end{tabular}

Teste exato de Fisher $(\mathrm{P}>0,005)$

média de 222,1 mmHg e não ocorreu ruptura dos cólons em nenhum dos animais do grupo II e em 5 (40,0\% ) do GI $(p<0,005)$. Desses, 3 (30\%) eram do subgrupo IA, e 2 (20,0\%) do GIB (P>0,005) (Tabela 4). Ocorreu ruptura da sutura em 4 ( $20 \%$ ) cães do grupo I, sendo 2 do subgrupo IA e 2 (20\%) do GIB com pressão média de 94,0 mmHg (Tabelas 5, 6 e 7). A análise histológica das amostras obtidas no $7^{\circ}$ d.p.o. demonstrou reprodução das fases do processo inflamaçãoreparação em ambos acessos utilizados. Para análise estatística, foram utilizados o teste Qui-quadrado corrigido de Yates para análise de complicações comparando o grupo I com o II, com $\mathrm{p}<0,005$ e o teste exato de Fisher para complicações comparando os subgrupos IA com GIB e da pressão máxima de tensão comparando os grupos I e II com $\mathrm{P}>0,005$.

TABELA 4 - Percentual de ruptura de sutura durante o teste de tensão nos grupos I e II

\begin{tabular}{llllllll}
\hline Grupo & \multicolumn{3}{c}{ Ruptura da Sutura } & \multicolumn{2}{l}{ Total } \\
& \multicolumn{2}{c}{ Presente } & \multicolumn{2}{c}{ Ausente } & \multicolumn{2}{l}{ No. \% } \\
& No. & $\%$ & No. & $\%$ & \multicolumn{2}{l}{ No. } \\
\hline I & 4 & 44,5 & 5 & 54,4 & 9 & 100 \\
II & - & - & 20 & 100 & 20 & 100 \\
\hline
\end{tabular}

Teste exato de Fisher $(\mathrm{P}<0,005)$

TABELA 5 - Percentual de ruptura de sutura durante o teste de tensão nos grupos IA e IB

\begin{tabular}{|c|c|c|c|c|c|c|}
\hline \multirow[t]{3}{*}{ Grupo } & \multicolumn{4}{|c|}{ Ruptura da Sutura } & \multicolumn{2}{|c|}{ Total } \\
\hline & \multicolumn{2}{|c|}{ Presente } & \multicolumn{2}{|c|}{ Ausente } & & \\
\hline & No. & $\%$ & No & $\%$ & \multicolumn{2}{|c|}{ No. \% } \\
\hline IA & 2 & 40 & 3 & 60 & 5 & 100 \\
\hline $\mathbb{B}$ & 2 & 50 & 2 & 50 & 4 & 100 \\
\hline
\end{tabular}

Teste exato de Fisher $(\mathrm{P}>0,005)$
TABELA 6 - Pressões (mmHg) registradas no teste de tensão no $7^{\circ}$ p.o., nos subgrupos A e B do grupo I

\begin{tabular}{lll}
\hline & \multicolumn{2}{c}{ Grupo I } \\
& A & B \\
\hline Sutura íntegra & 220 & 230 \\
& 230 & 220 \\
& 230 & \\
Sutura tamponada & 40 & 110 \\
& 80 & 160 \\
\hline
\end{tabular}

TABELA 7 - Pressões (mmHg) registradas no teste de tensão no $7^{\circ}$ p.o., nos subgrupos A e B do grupo II

\begin{tabular}{lll}
\hline & \multicolumn{2}{c}{ Grupo II } \\
& A & B \\
\hline Sutura íntegra & 220 & 220 \\
& 220 & 230 \\
230 & 230 \\
220 & 220 \\
220 & 220 \\
220 & 230 \\
230 & 220 \\
220 & 220 \\
220 & 220 \\
220 & 220 \\
\hline
\end{tabular}

\section{Discussão}

O acesso vídeo-laparoscópico vem sendo utilizado cada vez mais no tratamento de afecções colorretais benignas $^{18-22}$ e malignas ${ }^{23-25}$. As ressecções colorretais são procedimentos mais complexos, exigindo treinamento mais intenso pois o cólon é um órgão que ocupa os quatro quadrantes da cavidade abdominal e os procedimentos consistem na mobilização ampla, ligaduras vasculares e anastomoses intestinais feitas sob visão bidimensional. As suturas e anastomoses têm sido realizadas com segurança e rapidez utilizando-se grampeadores mecânicos. Entretanto, o cirurgião deverá encontrar-se apto a realizar a endo-sutura em situações em que se faça necessária. E o treinamento deve ser realizado preferencialmente em animal de experimentação porquanto o domínio da técnica de endosutura requer longo processo de adestramento. Neste estudo, foi escolhido o cão mestiço como animal de experimentação por ser dócil, de fácil aquisição e manuseio, além de não oferecer dificuldades na manutenção durante o período pós-operatório. Além disso, o cólon esquerdo é semelhante ao do ser humano. Entretanto, ao contrário do que ocorre com os suínos ${ }^{11,13}$, a cavidade peritoneal do cão limita o acesso laparoscópico devido ao tamanho reduzido, sobretudo para a confecção dos nós cirúrgicos, daí terem sido necessários animais com peso acima de $15 \mathrm{Kg}$.

$\mathrm{O}$ animal foi posicionado em decúbito dorsal com inclinação lateral direita, facilitando a exposição do colon esquerdo e afastando lateralmente as alças do intestino delgado. Foram posicionados cinco trocartes, sendo um no flanco direito para colocação da ótica, dois à esquerda (no 
flanco e fossa ilíaca), para apreensão e exposição do cólon esquerdo e dois outros (fossa ilíaca direita e epigástrio), à esquerda da linha média, para colocação da pinça de apreensão e porta-agulha. A ótica utilizada deve ser de boa resolução e posicionada entre os instrumentos de trabalho. A forma de apreensão do colon esquerdo expondo-o e simultâneamante ocluindo-o foi de fundamental importância para evitar contaminação da cavidade peritoneal. Outras alternativas de apreensão e oclusão têm sido demonstradas por outros autores no intestino delgado pois Noel $^{11}$ e col utilizaram fio transfixando a parede abdominal e intestinal, possibilitando a inversão do delgado durante a sutura da parede posterior. Já Waninger ${ }^{13}$ e col utilizaram fio somente na extremidade proximal, suspendendo e ocluindo o intestino. Neste estudo, foram utilizadas duas formas de abertura do colon esquerdo com e sem utilização do bisturi elétrico para avaliar as dificuldades técnicas na ocorrência de sangramento transoperatório significativo. Entretanto, não foi evidenciado sangramento abundante nos grupos sem utilização do bísturi elétrico, sendo a hemostasia realizada por compressão com gaze cirúrgica possibiltando realizar a endo-sutura manual. Portanto, a evolução pósoperatória foi distinta observando-se complicações nos grupos que utilizaram bísturi elétrico, ocorrendo deiscência total em aproximadamente $50 \%$ dos animais operados tanto pelo acesso laparotômico quanto laparoscópico. Medeiros ${ }^{26}$ et al referiram que nas anastomoses realizadas em cólon de cães nas quais utilizaram o bísturi elétrico, ocorreu maior formação de tecido colágeno e maior orientação das fibras. Entretanto, outros constataram maior quantidade de tecido necrótico e retardo no processo de cicatrização $0^{27,28}$. Enquanto, Aydos $^{29}$ et al não referiram quaisquer diferenças no processo de cicatrização. Na realização da endo-sutura laparoscópica foi importante o perfeito sincronismo do auxiliar-câmera com o cirurgião, aproximando a ótica no momento de penetração da agulha nos tecidos e distanciando-a na confecção do nó cirurgico devido à limitação proporcionada pela visão bidimensional nos procedimentos laparoscópicos. O porta-agulha deve formar um ângulo de $90^{\circ}$ com a pinça auxiliar para facilitar a confecção do nó cirúrgico. A agulha apropriada para realização da endo-sutura é do tipo “endo-ski”, combinando a curvatura na ponta para penetrar melhor nos tecidos com o segmento reto para facilitar a apreensão e manuseio. Foi utilizado neste estudo o fio de polidioxanona 000 pois é absorvível e monofilamentar, apresentando absorção mais fácil por hidrólise, não requerendo mecanismo celular e provocando mínima reação inflamatória nos tecidos. Também possibilita ainda o fácil deslizamento através dos tecidos ${ }^{30-}$ 32. Para facilitar a confecção do nó cirúrgico, foi utilizado fio com comprimento médio de $12 \mathrm{~cm}$, possibilitando realizá-lo com rapidez e segurança, como tem sido demonstrado também por Waninger ${ }^{13}$ e col. Adotando-se estes critérios técnicos, qualquer tipo de sutura pode ser realizada pois a parede do colon é observada com grande nitidez, proporcionando fácil identificação de suas camadas. Neste trabalho, foi realizada sutura com pontos separados, extramucosa para possibilitar maior treinamento na confecção de nós cirúrgicos. Os princípios técnicos aplicados na sutura intestinal pelo acesso laparoscópico são semelhantes aos do laparotômico, como preparo adequado do intestino para evitar contaminação da cavidade peritoneal, preservação do suprimento sangüíneo e ausência de tensão na linha de sutura. Entretanto, a expansão das indicações e a dificuldade técnica inicial em realizar a endo-sutura laparoscópica estimulou o desenvolvimento de instrumental cirúrgico mais adequado para facilitar o aprendizado e sua utilização na prática cirúrgica. Inicialmente, foram desenvolvidos instrumentos para realizar endo-nós cirúrgicos (endo-loop, endo-stitch) ${ }^{33-35}$. À medida em que os cirurgiões se familiarizaram com as técnicas de endo-sutura manual, estes instrumentos passaram a ser pouco utilizados. Com relação ao tipo de acesso cirúrgico, não foi observada diferença quanto a integridade da sutura, complicações e ao teste de tensão. A diferença dos resultados ocorreu na forma de abertura do colon. Os grupos que utilizaram o bisturi elétrico apresentaram deiscência da sutura em aproximadamente 50 \%. Os animais evoluíram com diárreia (3 a 5 evacuações líquidas por dia ) e vômitos (1 a 3 episódios por dia). Ocorreu óbito entre o $4^{\circ}$. e $7^{\circ}$ dias devido 'a peritonite. Nos demais, a sutura apresentava-se íntegra ou tamponada com epiplon, apresentando boa evolução clínica a despeito do acesso utilizado. Com relação aos resultados do teste de tensão das suturas, houve semelhança em todos os animais que apresentavam sutura íntegra com pressão média de 222 mmHg. Ocorreu ruptura da sutura nos grupos operados com bisturi elétrico, sendo em $20 \%$ dos cães operados por cada um dos acessos laparotômico ou laparoscópico cuja pressão média foi $94,0 \mathrm{mmHg}$. A segurança da sutura laparoscópica foi constatada através do teste de tensão, pois foi obtida a mesma pressão nos animais operados por laparotomia, também semelhante às pressões registradas no cólon normal. A análise histopatológica da zona de sutura comparando os acessos laparotômico e laparoscópico, demonstrou semelhança nas fases do processo de inflamação-reparação.

\section{Conclusão}

A endo-sutura manual laparoscópica em cólon de cães pode ser utilizada pois apresentou os mesmos resultados do acesso laparotômico. Os resultados não dependem do acesso cirúrgico escolhido mas do treinamento adequado e específico do cirurgião em vídeo-laparoscopia.

\section{Referências}

1. Saclarides TJ, Ko ST, Airan M, et al. Laparoscopic removal of a large colonic lipoma. Report of a case. Dis Colon Rectum. 1991; 34 (11): 1027-9.

2. Schlinkert RT. Laparoscopic-assisted right hemicolectomy. Dis Colon Rectum. 1991; 34 (11) : 1030-1031.

3. Fowler DL \& White SA. Laparoscopy assisted sigmoid resection. Surg Laparosc Endosc. 1991; 1 (3): 183-88.

4. Cooperman AM, Katz V, Zimmon D et al. Laparoscopic colon resection: a case report. J Laparoend Surg. 1991; 1(4): 221-4.

5. Jacobs M, Verdeja JC, Goldstein HS. Minimally invasive colon resection (laparoscopic colectomy). Surg Laparosc Endosc. 1991; 1 (3): 144- 50.

6. Phillips ED, Franklin M, Carrol BJ et al. Laparoscopic colectomy. Surg Laparosc Endosc. 1991; 1(6): 703-7. 
7. Larach SW, Salomon MC, Williamson PR et al. Laparoscopic assisted abdominoperineal resection. Surg Laparosc Endosc. 1993; 3 (2): 115-8.

8. Pappas TN. Laparoscopic colectomy. The innovation continues. Ann Surg. 1992; 216 (6): 701-2.

9. Wexner Sf, Johansen OB. Laparoscopic bowel rection: Advantages and limitations. Ann Med 1992;24:105-10.

10. Regadas FSP, Nicodemo AM, Rodrigues LV et al. Anastomose colorretal por via laparoscópica. Apresentação de dois casos e descrição da técnica operatória. Rev Bras Coloproct. 1992;12(1):21-3.

11. Noel P, Fagot H, Fabre JM et al. Résection anastomose de l’intestin grêle par coelioscopie chez le porc. Étude expérimentale comparative entre anastomose mécanique et manuelle. Ann Chir. 1994; 48(10):921-29.

12. Olson KH, Bolos G, Lowe MC et al. Comparative study of open laparoscopic intracorporeal and laparoscopic assisted low anaterior resection and anastomosis in the pigs. Am J Surg. 1995;61:197-201.

13. Waninger J, Salm R, Imdahl A et al. Comparasion of laparoscopic handsewn suture techniques for experimental small-bowel anastomoses. Surg Laparosc Endosc. 1996; 6 (4): 282-89.

14. Mehdi A, Closset J, Gay F et al. Laparoscopic treatment of a sigmoid perforation after colonoscopy. Case report and review of literature. Surg Endosc. 1996; 10: 666-67.

15. Reis Jr JA. Estudo comparativo entre a sutura manual e a mecânica no fechamento do coto retal por via vídeolaparoscópica. Tese de Mestrado. Escola Paulista Medicina, São Paulo, 1998.

16. Cushieri A, Szabo Z. Equipament and instrumentation for laparoscopic suturing In: Cushieri A., Szabo Z. Tissue aproximation in endoscopic surgery. Oxford: Ed. Isis Medical Media, 1995. p.95-112.

17. Sena JIN, Silva Filho AR, Penha GTC. Insuflador alternativo para Cirurgia Videoendoscópica Experimental. Rev Col Bras Cir. 1999; 25(6) :191-3.

18. Regadas FSP, Rodrigues LV, Nicodemo AM et al. Intervenções colorretais videolaparoscópicas. Experiência de 102 casos. Rev Bras Coloproctol. 1995; 15(3):110-3.

19. Sousa SJ, Martina FA, Carmel APW. Tratamento cirúrgico do megacólon chagásico por videolaparoscopia. In: Ramos JR, Regadas FSP, Sousa JS Cirurgia Colorretal por Videolparoscopia. Rio de Janeiro: Revinter, 1997. p.131-5.

20. Reis Neto JA, Quilici, FA, Cordeiro F et al. Cirurgia vídeolaparoscópica colorretal. Rev Bras Coloproctol. 1997; 7(4):234-8.
21. Regadas FSP, Regadas SMM, Rodrigues LV et al. Tratamento cirúrgico da doença diverticular através do acesso vídeo-laparoscópico. Apresentação de 35 casos. Rev Bras Coloproctol. 1998;18(1): 35-9.

22. Regadas FSP, Rodrigues LV, Regadas SMM et al. Sigmoidectomia laparoscópica no tratamento da doença diverticular hipertônica: experiência com 80 casos. Rev Bras Videocir. 2004; 2(1):14-18.

23. Leroy J, Jamali F, Forbel L et al. Laparoscopic total Mesorectal Excision (TME) for rectal cancer surgery: long-term outcomes. Surg Endosc 2004; 18(2):281-9

24. Regadas FSP, Regadas SMM, Rodrigues LV et al. Índices de recidiva e sobrevida no tratamento do câncer colorretal comparando os acessos laparoscópico e laparotômico em cinco anos. Rev Bras Coloproctol. 2001; 21(3):144-6

25. Reis Neto JA, Quilici FA, Cordeiro F et al. Laparoscopic total mesorectum excision. JSLS 2002; 6(2):163-7.

26. Medeiros RR, Vidal BC, Leonard SS. Emprego experimental do bísturi elétrico nas anastomoses do intestino grosso. Rev Bras Coloproctol. 1994; 4(4): 228-39.

27. Kott I, Lurie M. The effects of electrosurgery and the surgical knife on the healing of intestinal anastomoses. Dis Col Rectum. 1973; 16: 33-8.

28. Biondo-Simões, MLP, Sech M, Corbellini M et al. Estudo experimental comparando a cicatrização de anastomoses do cólon esquerdo após a diérese com bisturi elétrico e com bisturi frio. Rev Bras Coloproctol. 1997; 17(3): 186-90.

29. Aydos RD, Magalhães AM, Menzes Filho JF et al. Efeitos da diatermia cirúrgica na cicatrização de anastomoses do intestino grosso: Estudo experimental em coelhos. Acta Cir Bras. 1994; 9(4): 190-4.

30. Sanz LE, Paterson JA, Kamth R et al. Comparision of maaxon suture with vicryl, chromic catgut and PDS sutures in fascial closure in rats Obstet Gynecol. 1988; $17(3): 418-22$.

31. Bennett R. Continuing medical education: selection of wound closure materials. J Am Acad Dermatol. 1988; 18(4):619

32. Bourne RB, Bitar H, Andrea PR et al. In-vivo comparision of four absorbable sutures: vicryl, dexon, maxon and PDS. Can J Surg. 1988; 31(1):43-5.

33. Al Fallouji M. Making loops in laparoscoic surgery: State of the art. Surg Laparosc Endosc. 1993; 3(6):477-81.

34. Sedlack JD, Williams VM, Desimone J et al. Laparoscopic knot security. Surg Laparosc Endosc. 1993;6 (2):144-146.

35. Medina M. Introduction of a new double-looped suture. Surg Laparosc Endosc. 1995; 5(1):50-5.

\section{Correspondência}

Sthela Maria Murad Regadas

Av. Edilson Brasil Soares, 1892

60834-220 - Fortaleza - CE
Conflito de interesse: nenhum Fonte de financiamento: nenhuma

Recebimento: 21/03/2005 Revisão: 19/04/2005

Aprovação: 23/05/2005

\section{Como citar este artigo:}

Regadas SMM, Regadas FSP, Rodrigues LV, Carvalho CGSM, Regadas Filho FSP. Modelo experimental de sutura manual em colon de cão por vídeo-laparoscopia. Acta Cir Bras. [periódico na internet] 2005 Jul-Ago;20(4). Disponível em URL: http://www.scielo.br/acb 